\title{
openheart A hundred heart failure deaths: lessons learnt from the Dr Foster heart failure hospital mortality alert
}

\author{
Patrick Tran, ${ }^{\oplus 1}$ Michelle McDonald, ${ }^{2}$ Lleika Kunaselan, ${ }^{2}$ Fraz Umar, ${ }^{1}$ \\ Prithwish Banerjee ${ }^{\odot, 2,3}$
}

To cite: Tran P, McDonald M, Kunaselan L, et al. A hundred heart failure deaths: lessons learnt from the Dr Foster heart failure hospital mortality alert. Open Heart 2019;6:e000970. doi:10.1136/

openhrt-2018-000970

Received 20 November 2018 Revised 26 January 2019 Accepted 16 February 2019

Check for updates

(c) Author(s) (or their employer(s)) 2019. Re-use permitted under CC BY-NC. No commercial re-use. See rights and permissions. Published by BMJ.

${ }^{1}$ Department of Cardiology, University Hospitals Coventry \& Warwickshire, Coventry, United Kingdom

${ }^{2}$ Warwick Medical School, Coventry, United Kingdom ${ }^{3}$ CIRAL, Coventry University, Coventry, United Kingdom

Correspondence to Prof Prithwish Banerjee; Prithwish.Banerjee@uhcw. nhs.uk

\section{ABSTRACT}

Background Despite advances in evidence-based pharmacotherapy, the latest National Heart Failure Audit (NHFA) has shown that in-hospital mortality of heart failure (HF) remains high with large interhospital variations. University Hospitals Coventry \& Warwickshire, a tertiary cardiac centre, received a mortality alert of excess HF deaths based on a high Dr Foster hospital standardised mortality ratio (HSMR). This conflicted with our local NHFA data which showed lower than national average mortality rates.

Objective To review various systemic and individual processes of care in patients admitted with $\mathrm{HF}$ and examine the validity of HSMR in HF.

Design, setting, patients A retrospective case note analysis was performed on a random sample of $100 \mathrm{HF}$ deaths identified by Dr Foster from 2010 to 2016. Measures Case record reviews were performed on the following aspects of care: admission to appropriate wards, resuscitation status, palliative care input and National Confidential Enquiry into Patient Outcome and Death classification. Primary diagnosis coding, diagnostic accuracy and actual causes of death were examined to assess limitations of HSMR.

Results Despite evidence of lower mortality on cardiology wards, only $28 \%$ of patients with acute HF were admitted to a cardiology-ward. Sixty four per cent were considered palliative but only $4.6 \%$ were referred to palliative care. The Do Not Attempt Resuscitation order was appropriate in $91 \%$ patients but only $74 \%$ had this in place. The primary diagnosis of HF was incorrectly coded in $34 \%$ while three cases were misdiagnosed.

Conclusion HF may be coded as a cause of death in some cases where the cause is uncertain and misdiagnosed. Although HSMR has many limitations, it is a smoke alarm that should not be ignored.

\section{INTRODUCTION}

Heart failure (HF) is a leading cause of unplanned admissions and mortality in people aged 65 and above. ${ }^{1}$ Over the last decade since the inception of the National Heart Failure Audit (NHFA) in England and Wales, in-hospital mortality of HF has considerably improved. From $11.1 \%$ in $2011-2012$, the average mortality rate has fallen to $8.9 \%$

\section{Key messages}

What is already known about this subject?

- Dr Foster mortality alerts and their investigations in response to the alerts have been described in various areas of medicine from different hospitals.

- The concept of hospital standardised mortality ratios (HSMR) is generally well known, as well as the issue of clinical miscoding being extensively highlighted in the literature.

What does this study add?

- This study looks at a fairly large number of deaths by focusing on several aspects of heart failure (HF) hospital care in a structured manner such as admission to appropriate wards, resuscitation status, palliative care (PC) and National Confidential Enquiry into Patient Outcome and Death (NCEPOD) classification.

- Clinical coding issues surrounding HF deaths such as primary diagnosis and diagnostic accuracy were examined to demonstrate the limitations of HSMR use in $\mathrm{HF}$.

How might this impact on clinical practice?

- The results of our analysis highlight the status of HSMR in alerting every HF service of major fluctuations in its quality and performance.

- It brings focus onto PC, comorbidities, the shortcoming of the NCEPOD grading system and coding issues in its entirety.

- While recognising the complexity in assessing HF deaths, this study suggests the need for all HF services to be sensitive to HSMR data but not dependent on it. All of these are likely to improve services across the board.

in $2015-2016^{2}$. This is reflected by greater implementation of evidence-based care processes, such as providing specialist $\mathrm{HF}$ input on cardiology wards and initiating betablockers and ACE inhibitors in patients with HF with reduced ejection fraction (HFREF), recommended by National Institute for Health and Clinical Excellence (NICE). ${ }^{12}$ However, as highlighted in NHFA, there are still large variations in adherence to these 
standards among hospitals associated with wide differences in mortality. ${ }^{2}$ Obviously, survival rate is dependent on case-mix. ${ }^{3}$ Hospitals with older patient populations linked with multimorbidity, palliation and preponderance of HF with preserved ejection fraction (HFPEF) (which lacks prognostically beneficial therapies with a higher non-cardiovascular death incidence than HFREF) can be expected to have higher mortality. ${ }^{4-6}$ Therefore, as a comparative measure of performance, NHS Trusts employ risk-adjusted metrics such as the hospital standardised mortality ratios (HSMR) - a ratio of actual deaths to expected deaths multiplied by $100 .^{7}$ This is calculated by external benchmarking companies like Dr Foster Intelligence (DFI) ${ }^{8}$

University Hospitals Coventry and Warwickshire (UHCW) NHS trust is a tertiary centre that delivers specialist HF service and uses DFI to monitor its HSMR for HF annually. We were notified of a higher than expected HSMR in the financial year 2013-2014. Hence, to better understand the systemic and individual processes of care in these patients, we performed a retrospective case note review on a random sample of 100 patients from 2010 to 16 who were identified by DFI as in-hospital deaths with a primary diagnosis of HF.

We also looked at HF deaths from a different angle. We sought to determine whether the HSMR of HF in 20132014 (calculated by Dr Foster) truly reflected deaths of patients with a primary diagnosis of HF (ie, excluding misdiagnosed cases and other primary diagnoses) and finally, we aimed to evaluate the validity of HSMR as a quality indicator of care.

\section{METHODS}

\section{Setting and sample selection}

The study site is a tertiary-care hospital which delivers specialist HF service and receives a large number of emergency admissions for acute HF. The Data Quality Coding team collects data for coding in mortality and reviews the coding for accuracy. All deaths eventually coded with a primary diagnosis of $\mathrm{HF}$ and palliative care $(\mathrm{PC})$ coding are stored on the Performance and Programme Management Office database, which is analysed monthly before reporting to the trust's mortality review facilitator for finalisation. The concluding number of mortality cases is collected and monitored by DFI. All new mortality alerts are reported to the mortality review committee who assigns a clinical lead for investigation of the alert. The investigation into 2013-2014 HSMR alert revealed discrepancies in coding accuracy, diagnosis of $\mathrm{HF}$ and other confounding variables. To better understand this and appreciate any recurring themes and track changes over time in the care processes of HF, we decided to randomly select 100 cases from the DFI mortality database between January 2010 and December for a representative retrospective case-note review.

\section{Review process}

For the first part of analysis, we focused our review on the following aspects of care during the index admission leading up to death:

1. Admission to an appropriate ward.

2. Documentation of resuscitation status.

3. Palliative care input.

4. National Confidential Enquiry into Patient Outcome and Death (NCEPOD) classification of overall care.

For the second part of the analysis, the primary coding of death and actual cause of death were determined by reviewing the entire case notes, death certificate summaries which are attached by the bereavement office and the Clinical Results Reporting System (CRRS) database which contains treatment history, cardiology clinic and discharge letters and recent echocardiography reports. Each case was independently reviewed by two cardiology specialist registrars within the cardiology department. For each patient, a case vignette of the care leading up to death was created. The lead investigator, a senior cardiologist who leads the specialist HF service, performed an oversight of the review to ensure a uniform practice of the review process and identify any discrepancies. Any uncertainties, for example, the diagnosis of HF or cause of death, was discussed between the reviewers before reaching a group consensus. Disagreements with NCEPOD grading of cases, mostly when regrading below A, were discussed with the lead investigator for reanalysis of the case notes according to objective questions adapted from the Global Trigger Tool for measuring adverse events (Institute for Healthcare Improvement 2003) ${ }^{9}$ set out in the trust's mortality review electronic form.

\section{RESULTS}

\section{Admission to an appropriate ward}

Independent of age and disease severity, managing HF on a cardiology ward affords better survival for patients in hospital and 1 year postdischarge; they are more likely to receive up-to-date echocardiography and on finding HFREF, more likely to receive disease-modifying therapies. ${ }^{21011}$ In comparison, even after adjusting for age and comorbidities, patients with decompensated HF treated on general medical wards have a $59 \%$ increased risk of in-hospital death (HR 1.59 from 2010 to 2016, CI 1.54 to $1.65, \mathrm{p}<0.001) .^{2}$ Patients with more advanced HF on cardiology wards may be more likely to be considered for cardiac resynchronisation therapy or implantable cardioverter defibrillators as primary or secondary prevention, than those treated on general medical wards. ${ }^{10} 11$ According to the NHFA, $46 \%$ of patients were admitted to cardiology: static in the last 3 years ${ }^{2}$. At UHCW, this figure was $28 \%$ (table 1). Of patients not admitted to cardiology, $15 \%$ should have been (eg, for outliers on gynaecology and neurology), while the remaining had overarching medical needs that were best provided by another specialty. For example, patients with complex airways disease on non-invasive ventilation were appropriately transferred 


\begin{tabular}{ll}
\hline Table 1 Place of care by specialty \\
\hline Ward & $\begin{array}{l}\text { Percentage of patients } \\
(\mathbf{n}=\mathbf{1 0 0 )}\end{array}$ \\
\hline Cardiology & 28 \\
\hline Respiratory & 22 \\
\hline Gerontology & 12 \\
\hline Neurology & 11 \\
\hline Acute Medical Unit & 10 \\
\hline Critical Care Unit & 6 \\
\hline General Medicine & 3 \\
\hline Renal & 3 \\
\hline Gynaecology & 3 \\
\hline Oncology & 2 \\
\hline
\end{tabular}

to the respiratory ward, and cases of advanced dementia and neurodegenerative disease were managed on geriatric units. From individual case descriptions, patients on non-cardiology wards tended to have more documentations of 'frailty' and 'cachexia'; however, specific data for comparison with those on cardiology wards were not collected. Nonetheless, frailty is associated with worse outcomes and higher mortality in elderly patients with $\mathrm{HF}^{12}$ Despite the place of care, patients can be referred to the HF specialist team to review and support the HF management. This outreach service, known to improve mortality, was delivered to $33 \%$ of UHCW patients in non-cardiology settings, which is better than the national figure of $24 \%$ reported in the latest NHFA. ${ }^{2}$ For equity of specialist care irrespective of ward allocation, UHCW's HF specialist team has expanded over the last 3 years and integrated with the electronic referral system to meet this increasing demand which seems to be having a positive impact.

\section{Resuscitation status}

Similar to the NHFA, the median age of our sample population was around 84 years (figure 1), risen from 78 years in 2008. ${ }^{2}{ }^{13}$ This progressively ageing population constitutes the majority of HF admissions and with higher prevalence of HFPEF, comorbidity burden and frailty carries a higher risk of mortality. ${ }^{5}$ Accordingly, resuscitation status needs to be reviewed early and regularly, especially given

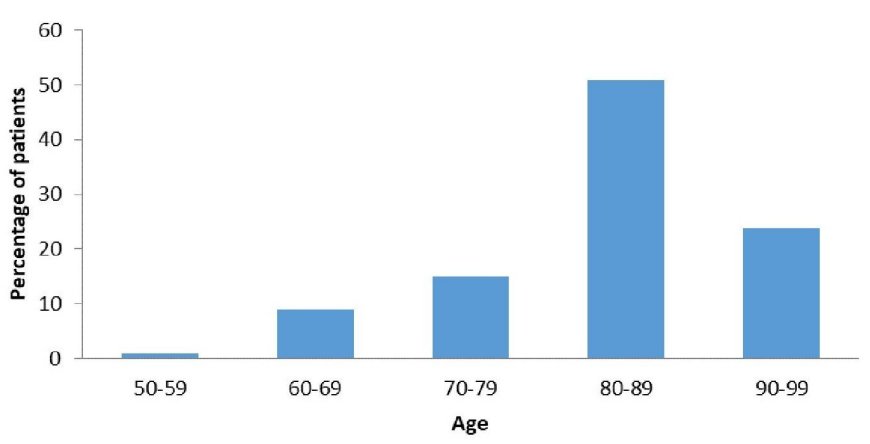

Figure 1 Age distribution of patients. the unpredictable clinical trajectory of HF. ${ }^{14}$ If measures such as cardiopulmonary resuscitation (CPR) are anticipated to prolong suffering and deemed futile, a Do Not Attempt CPR (DNACPR) decision should be clearly communicated and documented. Of the 100 patients reviewed, DNACPR was appropriate in $91 \%$ but only $74 \%$ had this in place owing to inconsistencies in communication and documentation. In these instances, DNACPR was either written as a management plan but awaiting family discussion or verbally agreed in family meetings without completing the DNACPR form. In some cases, this resulted in inappropriate CPR attempts.

Barriers to resuscitation discussions often come down to communication and misunderstanding. Patients may misconceive $\mathrm{HF}$ as a benign condition and even at end-stages, may pursue aggressive life-prolonging therapies which clinicians may accept to avoid conflict or despondence. ${ }^{15}$ Withdrawal of treatment such as deactivating ICDs is one example. ${ }^{16}$ Doctors and nurses may mix DNACPR with end-of-life care, denying patients the opportunity of active treatment. ${ }^{15}$ In one observational study, patients with DNACPRs were less likely to have echocardiograms or receive disease-modifying therapy than their counterparts. ${ }^{17}$ To overcome these obstacles, UHCW has established the Recommended Summary plan for Emergency Care and Treatment (ReSPECT), replacing DNACPR forms (figure 2). ReSPECT provides a decision support framework for clinicians to form a tailored patient-centred plan on emergency treatments, including CPR, for all patients regardless of their functional status. It contextualises resuscitation within a patient's broader goals of care. ${ }^{18}$

\section{Palliative care}

One important lesson from the mortality alert is to look at our PC services. For some patients, the treatment goal is palliation where the focus is not on improving survival but on quality of life, and early identification and referral is a key NICE recommendation monitored by NHFA. ${ }^{12} 19$ Nationally, only $4 \%$ of patients were formally referred to PC. $^{2}$ Equally in our sample, $64 \%$ patients were considered palliative but only $4.6 \%$ were referred (mostly when death was imminent) to the PC team. The issue does not lie in prescribing palliative medications, which clinicians do well, but rather acknowledging the need for $\mathrm{PC}$ and communicating this to patients. ${ }^{20}$ In a primary and tertiary care survey of clinicians including cardiologists, $30 \%$ reported a lack of confidence in discussing or providing end-of-life care. Some felt that this responsibility rests with the PC team. ${ }^{21}$ In an effort to improve this, the latest European Society of Cardiology HF guidelines has included guidance on PC. For example, it describes warning signs that should trigger consideration for palliation, for example, cardiac cachexia or recurrent exacerbations within 6 months despite optimal tolerated therapy. ${ }^{22}$ Over the last year at UHCW, PC has had a more recognised and integral role in the HF services; supporting fast-track discharges of patients who prefer 


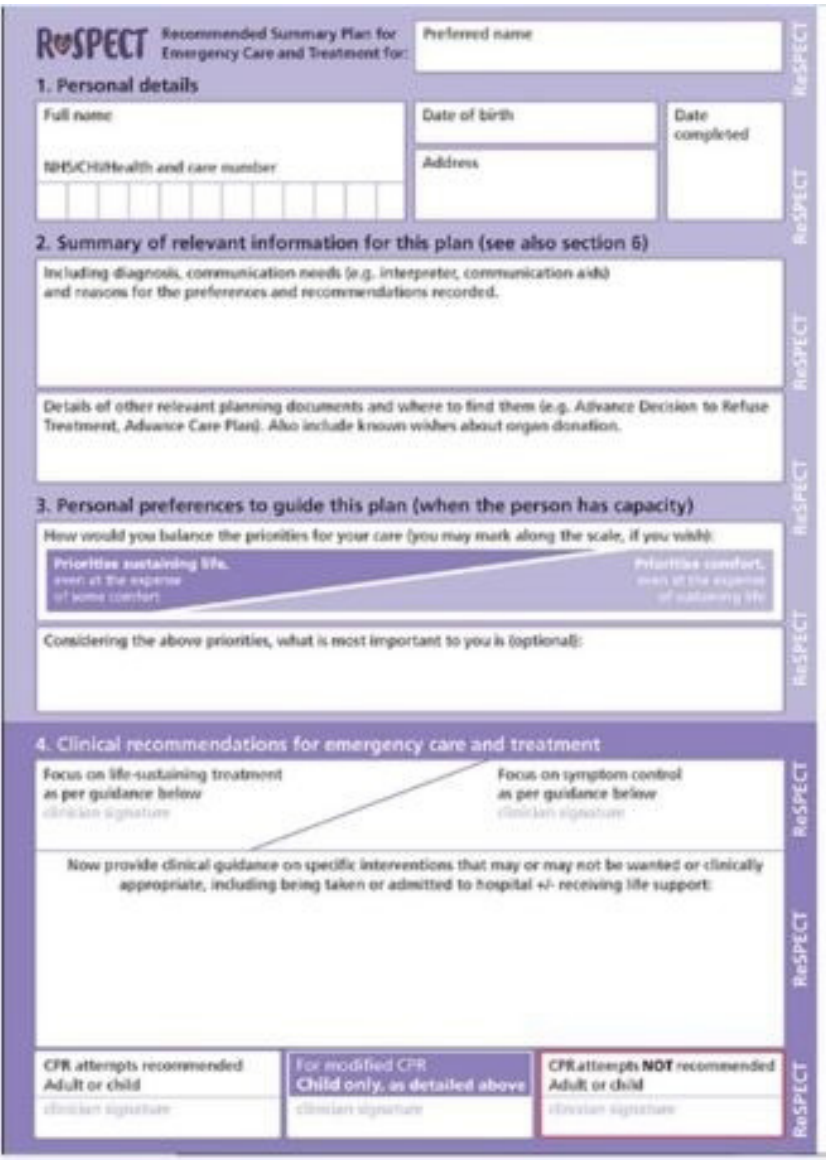

5. Capacity and representation at time of completion

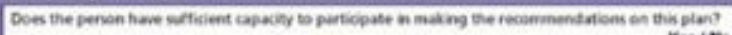

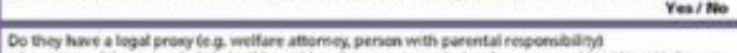

Nen/ No/Uninement

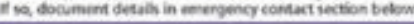

6. Involvement in making this plan

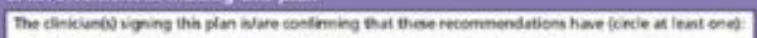

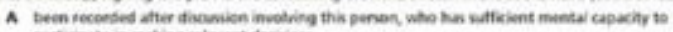

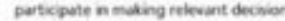

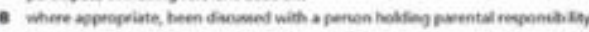

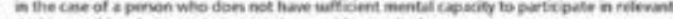

decibion mading been made in wewerdence with capsily las

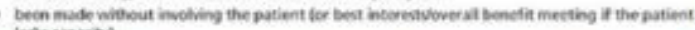
lasiscaparing

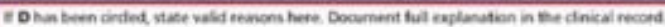

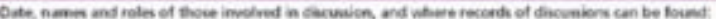

\section{Cinikians' vionatures}

\begin{tabular}{|c|c|c|c|c|}
\hline Devopution & Cinidan namb & 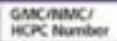 & Sonatuent & Date s time \\
\hline & & & & \\
\hline & & & & \\
\hline
\end{tabular}

Senior renomith clonicion

8. Emergency contacts

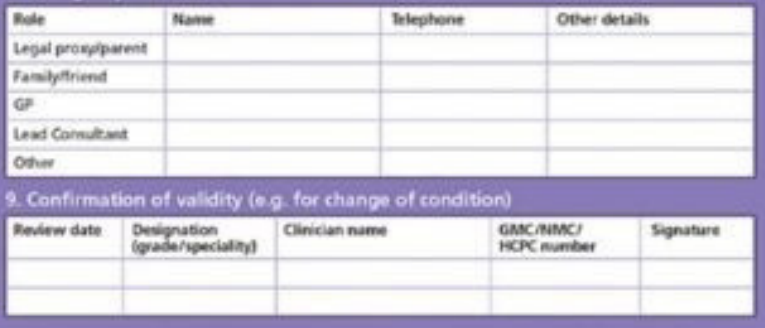

\section{DO NOT ATTEMPT CARDIOPULMONARY RESUSCITATION} Adeula aged 16 years and ever

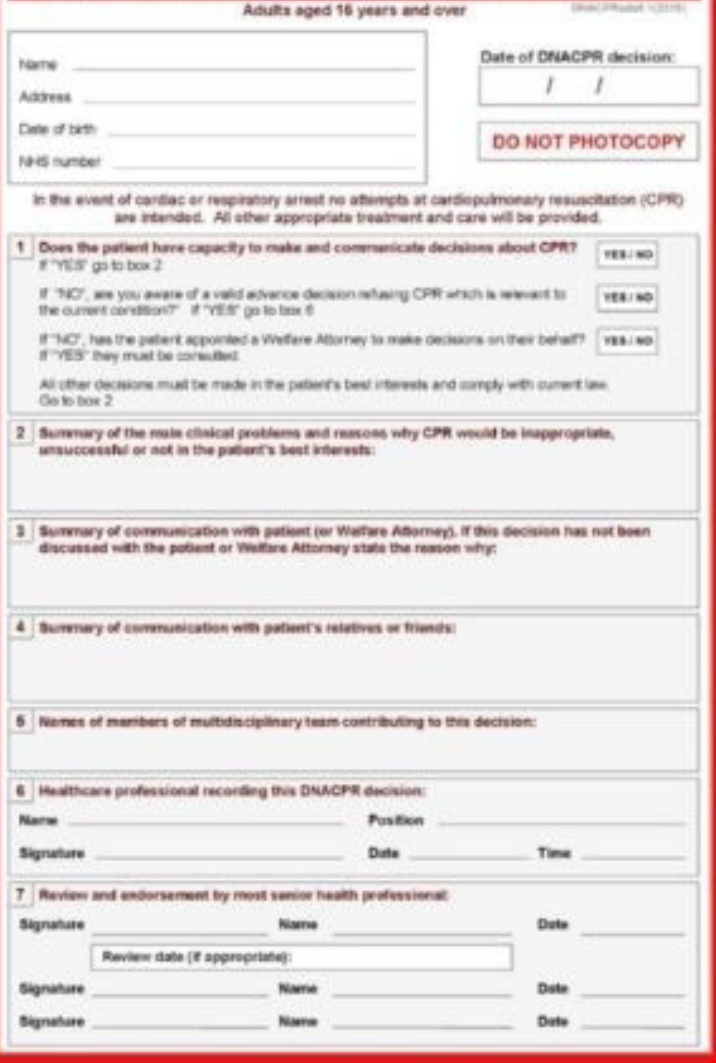

Figure 2 ReSPECT Form (top) which has replaced the DNACpR form (bottom) at UHCW. 


\begin{tabular}{|c|c|}
\hline NCEPOD Grade & $\begin{array}{l}\text { Percentage of } \\
\text { patients }(n=100)\end{array}$ \\
\hline Grade A (Good practice) & 87 \\
\hline Grade B (Concerns with aspects of clinical care) & 10 \\
\hline $\begin{array}{l}\text { Grade } \mathrm{C} \text { (Concerns with aspects of } \\
\text { organisational care) }\end{array}$ & 1 \\
\hline $\begin{array}{l}\text { Grade D (Concerns with aspects of both clinical } \\
\text { and organisational care) }\end{array}$ & 2 \\
\hline $\begin{array}{l}\text { Grade } E \text { (Concerns with several aspects of } \\
\text { clinical and/or organisational care) }\end{array}$ & 0 \\
\hline
\end{tabular}

NCEPOD, National Confidential Enquiry into Patient Outcome and Death.

to die at home and providing ethical guidance on withdrawal of life-sustaining therapies.

\section{NCEPOD grading}

UHCW has an extensive mortality review system to assess the quality of care in patients before death and cases graded below NCEPOD grade A undergo in-depth secondary reviews by another consultant. The spread of NCEPOD classifications of our sample population is shown in table 2, which also explains the grades from A to E. Several recurring themes were identified in cases graded B-D and these echoed the aspects of care reviewed above. These include inappropriate ward allocation, no referral to PC and absence of DNACpR leading to inappropriate CPR. Notably, patients graded as $\mathrm{D}$ often related to admissions to inappropriate wards without outreach cardiology input, reflecting a deficit in both clinical and organisational care.

Following our review of the deaths for appropriateness of the allotted grading, $6 \%$ of grade A cases were felt to be actually at the lower grade $\mathrm{B}$ for reasons similar to above: no referral to $\mathrm{PC}$ and inappropriate place of care. One case was regraded to $\mathrm{C}$ due to a delayed discharge linked with multiple hospital-acquired infections. Primary mortality reviews at this time were carried out by the consultant responsible for the patient's care; however as illustrated, this can sometimes introduce a degree of bias and hinder opportunities for further improvement. Learning from this and similar retrospective reviews done in the hospital, the practice has now changed to the primary mortality reviews now being done by a consultant not responsible for the patient's care during that admission.

\section{Limitations of HSMR}

In 2013-2014, DFI identified 107 patients who died with a primary diagnosis of HF at UHCW. DFI calculated the expected deaths at 81.42 and HSMR at 131, triggering a mortality risk alert. A high HSMR has huge reputational and financial implications on a hospital so it is crucial that the patient administrative database on which the actual deaths is based and variables that affect the expected deaths are as accurate and complete as possible. ${ }^{23}$ Observed deaths (the numerator of HSMR) will be influenced by correct diagnosis of HF and coding of primary diagnosis while the expected deaths (denominator of HSMR) will depend on demographics, comorbidities, severity of HF and palliative status as well as immeasurable case-mix factors. Many studies have highlighted limitations with HSMR and even argued against its use. ${ }^{23-25}$ In a case-note review of 86 patients (out of 107 ) in 2013-2014, we identified some practical shortcomings and caveats in interpreting HSMR in HF.

\section{Coding the primary diagnosis}

Inaccurate clinical coding is a well-known universal problem but the particular clinical coding problems for HF deaths is less familiar. HSMR is based on the primary diagnosis: the condition that led to hospital admission. ${ }^{8}$ For instance, all patients admitted with HF who die regardless of the cause, for example, pneumonia are coded as HF deaths, while patients admitted for another reason but die from HF should not be included. Retrospective case note analysis offered the reviewers the benefit of having the full clinical picture from admission to the date of death in order to differentiate the primary diagnoses from secondary diagnoses. For example, in one case where HF was initially suspected and coded as 'heart failure' as the primary diagnosis, a subsequent chest radiograph revealed evidence of pneumonia and was treated as such the following few days. Pneumonia would have been the primary diagnosis. In another example, a patient with known history of HF presented with legs swelling and ascites. This was initially treated as decompensated HF; however ensuing tests, for example, ascitic analysis, CT abdomen and blood cultures later revealed secondary peritonitis and liver disease. Altogether, we found that $34 \%$ of primary diagnosis were incorrectly coded as HF in our cohort as the actual primary diagnosis became clearer later on during admission (figure 3). Similarly, the NHFA 2013-2014 omitted almost 5000 patient records because the primary diagnosis was not $\mathrm{HF}$

\section{Primary Diagnosis $(n=86)$}
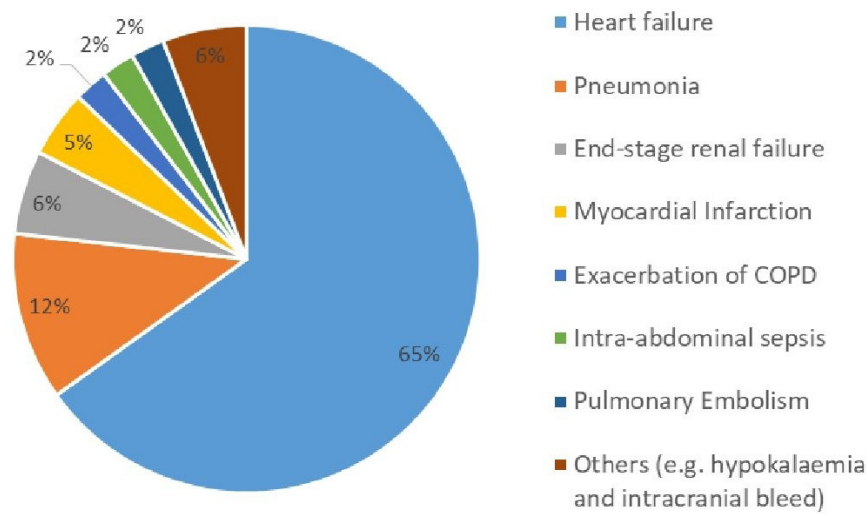

Figure 3 Breakdown of actual primary diagnoses leading to admission. 
despite being coded as such. ${ }^{26}$ A possible explanation was that the primary diagnosis only became apparent much later on but some clinical coders may only analyse the first few consultant episodes from the time of admission, which is often brief with important investigations still pending, for example, echocardiogram or B-type natriuretic peptide (BNP). During this period, patients may be provisionally treated and coded as HF (especially, if this was one of their comorbidities). In some patients, HF was a complication rather than the reason for admission (eg, decompensated HF from pneumonia). Furthermore, elderly patients often present with multiple problems, symptomatic comorbidities and organ failures; deciding which one should be the primary diagnosis is difficult for clinicians, let alone clinical coders.

\section{Diagnosis}

Correct diagnosis is another quality issue and can be challenging to establish in elderly patients because of the variable non-specific complaints (eg, fatigue and weakness) while some die quickly before BNP and echocardiogram are available. ${ }^{27}$ Occasionally, there may be alternative explanations for the symptoms but HF becomes a diagnostic scapegoat. ${ }^{28}$ In our review, $40 \%$ had BNP checked and $94 \%$ had echocardiography of which four who did not still had convincing evidence of HF clinically. Three patients were misdiagnosed. Even when HF is subsequently ruled out by cardiology, the coding of HF may remain in future discharge summaries. This can create errors in clinical coding and in prospective assessments of elderly patients with vague symptoms.

\section{Immeasurable confounding variables}

Just as important as proper diagnosis is attention to comorbidities. On average, patients with HF have 4.5 comorbidities and the depth of coding influences the Charlson comorbidity score which positively correlates with mortality. ${ }^{29-31}$ However, Charlson score only includes chronic conditions that were present before admission and does not include conditions that are newly identified during hospital stay, for example, undiagnosed Chronic Obstructive Airways Disease (COPD), thus potentially biasing the depth of coding and HSMR. ${ }^{24}$ Also, the former very much depends on the veracity of recorded comorbidities in clinical notes which is often incomplete.

Another problem is that the current ICD-10 coding for HF does not capture severity of HF and is only based on aetiology and pathophysiology. Since higher New York Heart Association classes are associated with higher mortality in both HFREF and HFPEF groups, ${ }^{32}$ it can be argued that a high HSMR in 1 year may be due to greater numbers of hospitalised patients with more severe or end-stage HF.

Clearly, patients admitted palliatively or treated on the end-of-life pathway are inherently more likely to die in hospital, regardless of the quality of hospital care. In 2004, the PC specialty code Z515 was introduced and was found to have a modest impact on HSMR. ${ }^{33}$ In our review, 16 patients were treated palliatively but none of them were PC coded. Since 2014, patients at UHCW who are seen by the PC team have stickers labelled 'Palliative Care Team' in their notes; this may help overcome undercoding of PC cases.

\section{Actual causes of death}

HSMR does not tell us the actual causes of death and in many NHS trusts including UHCW, coders do not have access to death certificates to ascertain this. It is striking that about a quarter of HF deaths are non-cardiac, with the most common being pneumonia, sepsis and malignancy ${ }^{3435}$ Patients with HF have double the risk of developing pneumonia whereby one mechanism suggests pulmonary congestion hampering respiratory microbial clearance. ${ }^{36}$ In our review, while the majority of deaths were due to cardiac causes ( $72 \%$ from $\mathrm{HF}$ and $6 \%$ from myocardial infarction), $21 \%$ were non-cardiac deaths which consisted of pneumonia $(10 \%)$, sepsis $(4 \%)$, pulmonary disease $(4 \%)$, gastrointestinal bleed $(2 \%)$ and malignancy $(2 \%)$. Non-cardiac conditions can increase mortality, precipitate and complicate HF admissions, and we therefore need to better recognise this when managing $\mathrm{HF}$ and interpreting the HSMR. ${ }^{34}$

\section{CONCLUSION AND IMPLICATIONS}

The original HSMR in 2013-2014 was 131. After excluding the 30 patients with an incorrect primary diagnosis and 3 with a misdiagnosis of HF, we calculated the HSMR at 68.8. Unlike HSMR which is based on patient administrative data, UHCW collects data for the NHFA using a stringent national pro-forma that ensures diagnosis is accurate and admission details are complete. The local mortality for HF at UHCW was $8.3 \%$ in 2014-2015 and $7.4 \%$ in 2015-2016 lower than the current national average of $8.9 \%$.

Our retrospective case-note review has given valuable insight into various processes of care for HF leading up to death. It has highlighted the importance of recognising PC and addressing non-cardiac comorbidities promptly. It has shown that although the majority of patients received good care, not all those with NCEPOD grade A are without criticism. From another angle, it has drawn attention to the difficulties in assessing and understanding HF deaths. Coding has a major influence on HSMR. Sometimes, HF may be coded as a cause of death in some cases where the cause is uncertain and misdiagnosed when presentation is vague and clouded by multimorbidity. Ultimately, HSMR has many limitations and is not ideal for inter-hospital comparisons but since no single indicator is perfect, it still provides a starting point for quality assessment and a smoke alarm that should not be ignored. Similarly, complacency based on acceptable HSMR may also not be justified. Recognition of the need for constant review of services in order to identify and correct deficiencies in the care provided is what defines a good HF service. 
Contributors PT, MM, LK and PB contributed to data collection, analysis and interpretation of data. PT drafted the article. PB initiated and led the project design and oversaw the data acquisition process. PB, FU and PT participated in revision of the manuscript.

Funding The authors have not declared a specific grant for this research from any funding agency in the public, commercial or not-for-profit sectors.

Competing interests None declared.

Patient consent for publication Not required.

Provenance and peer review Not commissioned; externally peer reviewed.

Data sharing statement All data relevant to the study are included in the article or uploaded as supplementary information.

Open access This is an open access article distributed in accordance with the Creative Commons Attribution Non Commercial (CC BY-NC 4.0) license, which permits others to distribute, remix, adapt, build upon this work non-commercially, and license their derivative works on different terms, provided the original work is properly cited, appropriate credit is given, any changes made indicated, and the use is non-commercial. See: http://creativecommons.org/licenses/by-nc/4.0/.

\section{REFERENCES}

1. National Institute for Health and Care Excellence (NICE). Acute heart failure: diagnosis and management (CG187), 2014. Available: https:// www.nice.org.uk/guidance/cg187 [Accessed Dec 2017].

2. National Heart Failure Audit (NHFA). April 2015 - March 2016 National Institute for cardiovascular outcomes research (NICOR), Institute of cardiovascular science, University College London, 2016. Available: http://www.ucl.ac.uk/nicor/audits/heartfailure/documents/ annualreports/annual-report

3. Jarman B, Gault S, Alves B, et al. Explaining differences in English hospital death rates using routinely collected data. BMJ 1999;318:1515-20.

4. Abdelhafiz AH. Heart failure in older people: causes, diagnosis and treatment. Age Ageing 2002;31:29-36.

5. Kaila K, Haykowsky MJ, Thompson RB, et al. Heart failure with preserved ejection fraction in the elderly: Scope of the problem. Heart Fail Rev 2012;17:555-62.

6. Dunlay SM, Roger VL, Redfield MM. Epidemiology of heart failure with preserved ejection fraction. Nat Rev Cardiol 2017;14:591-602.

7. Shahian DM, Wolf RE, lezzoni LI, et al. Variability in the measurement of hospital-wide mortality rates. N Engl J Med 2010;363:2530-9.

8. Dr Foster Intelligence. Mortality measurement: the case in favour. 15 July 2014. Imperial College London, 2014. Available: http://www. drfoster.com/wp-content/uploads/2014/08/Mortality_Measurement the case_in_favour.pdf

9. Institute for Healthcare Improvement,. IHI global trigger tool for measuring adverse events, 2018. Available: http://www.ihi.org/ resources/Pages/Tools/IHIGlobalTriggerToolforMeasuringAEs.aspx [Accessed cited January 20th 2019].

10. Cowie MR, Anker SD, Cleland JGF, et al. Improving care for patients with acute heart failure: before, during and after hospitalization. ESC Heart Fail 2014;1:110-45.

11. Parmar KR, Xiu PY, Chowdhury MR, et al. In-hospital treatment and outcomes of heart failure in specialist and non-specialist services: a retrospective cohort study in the elderly. Open Heart 2015;2:e000095.

12. Murad K, Kitzman DW. Frailty and multiple comorbidities in the elderly patient with heart failure: implications for management. Heart Fail Rev 2012;17:581-8.

13. National Heart Failure Audit (NHFA) 2008 - 2009. National Institute for cardiovascular outcomes research (NICOR), Institute of cardiovascular science, University College London, 2016. Available: http://www.ucl.ac.uk/nicor/audits/heartfailure/documents/ annualreports/hfannual08-09.pdf
14. Tanvetyanon T, Leighton JC. Life-sustaining treatments in patients who died of chronic congestive heart failure compared with metastatic cancer. Crit Care Med 2003;31:60-4.

15. Fritz Z, Slowther A-M, Perkins GD. Resuscitation policy should focus on the patient, not the decision. BMJ 2017;356.

16. Chen-Scarabelli C, Saravolatz L, Hirsh B, et al. Dilemmas in endstage heart failure. J Geriatr Cardiol 2015;12:57-65.

17. Chen JLT, Sosnov J, Lessard D, et al. Impact of do-not-resuscitation orders on quality of care performance measures in patients hospitalized with acute heart failure. Am Heart J 2008;156:78-84.

18. Respect. Recommended summary plan for emergency care and treatment. Available: http://www.respectprocess.org.uk/ [Accessed 29 Dec 2017].

19. Gibbs JSR, McCoy ASM, Gibbs LME, et al. Living with and dying from heart failure: the role of palliative care. Heart 2002;88 Suppl 2.

20. Mcllvennan CK, Allen LA. Palliative care in patients with heart failure. BMJ 2016;353:i1010.

21. Dunlay SM, Foxen JL, Cole T, et al. A survey of clinician attitudes and self-reported practices regarding end-of-life care in heart failure. Palliat Med 2015;29:260-7.

22. McMurray JJV, Adamopoulos S, Anker SD, et al. ESC guidelines for the diagnosis and treatment of acute and chronic heart failure 2012: the task Force for the diagnosis and treatment of acute and chronic heart failure 2012 of the European Society of cardiology. developed in collaboration with the heart Failure Association (HFA) of the ESC. Eur Heart J 2012;33:1787-847.

23. Black N. Assessing the quality of hospitals. BMJ 2010;340:c2066.

24. Bottle A, Jarman B, Aylin P. Strengths and weaknesses of hospital standardised mortality ratios. BMJ 2010;342:c7116.

25. Lilford R, Pronovost P. Using hospital mortality rates to judge hospital performance: a bad idea that just won't go away. BMJ 2010;340:c2016.

26. National Heart Failure Audit (NHFA) April 2013 - March 2014. National Institute for cardiovascular outcomes research (NICOR), Institute of cardiovascular science, University College London, 2016. Available: http://www.ucl.ac.uk/nicor/audits/heartfailure/documents/ annualreports/hfannual13-14.pdf

27. Teixeira A, Parenica J, Park JJ, et al. Clinical presentation and outcome by age categories in acute heart failure: results from an international observational cohort. Eur J Heart Fail 2015;17:1114-23.

28. Valk MJ, Mosterd A, Broekhuizen BD, et al. Overdiagnosis of heart failure in primary care: a cross-sectional study. Br J Gen Pract 2016;66:e587-92.

29. Saczynski JS, Go AS, Magid DJ, et al. Patterns of comorbidity in older adults with heart failure: the cardiovascular research network preserve study. J Am Geriatr Soc 2013;61:26-33.

30. Charlson ME, Pompei P, Ales KL, et al. A new method of classifying prognostic comorbidity in longitudinal studies: development and validation. J Chronic Dis 1987:40:373-83.

31. Oudejans I, Mosterd A, Zuithoff NP, et al. Comorbidity drives mortality in newly diagnosed heart failure: a study among geriatric outpatients. J Card Fail 2012;18:47-52.

32. Dalos D, Mascherbauer J, Zotter-Tufaro C, et al. Functional status, pulmonary artery pressure, and clinical outcomes in heart failure with preserved ejection fraction. J Am Coll Cardiol 2016;68:189-99.

33. Fekri O, Amuah JE, Herasimovich V, et al. Palliative care coding practices in Canada since the introduction of guidelines and the HSMR indicator. BMJ Open 2015;5:e008753.

34. Braunstein JB, Anderson GF, Gerstenblith G, et al. Noncardiac comorbidity increases preventable hospitalizations and mortality among Medicare beneficiaries with chronic heart failure. J Am Coll Cardiol 2003:42:1226-33.

35. Pons F, Lupón J, Urrutia A, et al. Mortality and cause of death in patients with heart failure: findings at a specialist multidisciplinary heart failure unit. Revista Española de Cardiología 2010;63:303-14

36. Mor A, Thomsen RW, Ulrichsen SP, et al. Chronic heart failure and risk of hospitalization with pneumonia: a population-based study. Eur J Intern Med 2013;24:349-53. 